\title{
Analysis of a projection/characteristic scheme for incompressible flow
}

\author{
Jean-Luc Guermond ${ }^{1, \dagger}$ and Peter D. Minev ${ }^{2, *, \ddagger}$ \\ ${ }^{1}$ LIMSI, CNRS-UPR 3152, BP 133, 91403 Orsay, France \\ ${ }^{2}$ Department of Mathematics and Statistical Science, University of Alberta, Edmonton, Canada T6G $2 G 1$
}

\begin{abstract}
SUMMARY
The paper presents the convergence analysis of a characteristic/projection scheme for the incompressible Navier-Stokes equations. This scheme is a modification of the scheme analysed in Achdou and Guermond (SIAM J. Numer. Anal. 2000; 37(3):799) which does not eliminate the projected velocity field from the system but rather uses it as the advecting field in the explicit characteristic advection. This field has a zero (generalized) divergence and is therefore more suitable for this purpose. It appears that this scheme has the same convergence rate as the one in Achdou and Guermond (SIAM J. Numer. Anal. 2000; 37(3):799) but on a given grid seems to produce more accurate results. The computational cost is not significantly higher since it requires only one extra inversion of the mass matrix which can be done relatively efficiently. We present numerical results which illustrate the properties of the scheme. Copyright (C) 2003 John Wiley \& Sons, Ltd.
\end{abstract}

KEY WORDS: projection methods; method of characteristics; finite-element method

\section{INTRODUCTION}

The projection methods have recently been combined with a characteristic method in a scheme proposed and analysed by Achdou and Guermond [1]. The idea seems somewhat odd at a first glance because projection methods usually produce an end-of-step velocity field whose divergence (although controlled) is relatively large, while the solenoidality of the advecting field is important for the stability of the method of characteristics. As proved in Reference [1], however, this combination does produce a convergent and very efficient algorithm. For a discussion on this issue and references to important papers on projection and characteristic methods the reader is referred to Achdou and Guermond [1] and the references therein.

\footnotetext{
*Correspondence to: P. Minev, Department of Mathematical Sciences, University of Alberta, Edmonton, Canada T6G 2G1.

†E-mail: guermond@limsi.fr

‡E-mail: minev@ualberta.ca

Contract/grant sponsor: Centre National de la Recherche Scientifique (CNRS)

Contract/grant sponsor: National Science and Engineering Research Council (NSERC)
} 
In Reference [2] a very similar scheme to the one introduced in Reference [1] is proposed. In Reference [1], the authors use the viscous velocity (the one obtained at the first substep) to perform the advection substep, whereas in Reference [2] the authors use the end-of-step velocity for this purpose. Even though in the discrete setting chosen in Reference [2], the end-of-step velocity is discontinuous and no obvious a priori bound on its divergence can be derived, it is reasonable to think that this velocity field may possibly be more suitable to use in the characteristic method than the viscous one since the projection step enforces on this field a constraint which is close to solenoidality. The goal of the present paper is to explore this idea. We study a first-order projection algorithm that uses the end-of-step velocity (projected onto the discrete space of continuous velocities) to perform the Lagrangian step of the algorithm. We show that the same error analysis as that in Reference [1] applies and yields the same stability criteria. Although the theoretical analysis does not show that the proposed strategy should perform better than the one in Reference [1], numerical results reported in Section 4 show that, in some cases, the proposed scheme seems to give better results.

The remainder of the paper is organized as follows. Section 2 recalls some basic stability and interpolation results concerning the characteristic and projection methods. Section 3 presents the modified scheme together with its convergence analysis. Finally, to illustrate the features of the modified scheme, Section 4 shows some numerical results on benchmark problems.

\section{PRELIMINARY RESULTS}

\subsection{Navier-Stokes equations and their spatial discretization}

We consider the unsteady Navier-Stokes problem

$$
\begin{aligned}
& \frac{\partial \mathbf{u}}{\partial t}+(\mathbf{u} \cdot \nabla) \mathbf{u}=-\nabla p+\frac{1}{R e} \nabla^{2} \mathbf{u} \quad \text { in } \Omega \times(0, T) \\
& \nabla \cdot \mathbf{u}=0 \quad \text { in } \Omega \times(0, T) \\
& \mathbf{u}=0 \quad \text { on } \partial \Omega \times(0, T), \quad \mathbf{u}=\mathbf{u}_{0} \quad \text { on } \Omega \times\{0\}
\end{aligned}
$$

where $\Omega$ is a smooth open bounded connected domain in $\mathbb{R}^{d}, d=2$ or 3 . For the sake of simplicity we consider only homogeneous Dirichlet conditions for the convergence analysis, but other boundary conditions can be considered and are actually used in the numerical simulations reported in Section 4.

The standard Lebesgue spaces are denoted by $L^{p}$. We denote the usual Sobolev spaces by $W^{s, p}(\Omega), 0 \leqslant s \leqslant \infty, 0 \leqslant p \leqslant \infty$ and the corresponding norms and semi-norms by $\|\cdot\|_{s, p}$, $|\cdot|_{s, p}$. For $p=2$ we set $H^{s}=W^{s, 2}$ and the related norm (semi-norm) is denoted by $\|\cdot\|_{s}\left(|\cdot|_{s}\right.$ ). Sobolev and Lebesgue spaces with a zero subscript are the completion of the space of the smooth functions compactly supported in $\Omega$. We finally introduce $V=\left\{\mathbf{v} \in H_{0}^{1}(\Omega)^{d}, \nabla \cdot \mathbf{v}=0\right\}$. Throughout the paper we use $c$ to denote a generic constant that depends only on the data of the problem, that is, $c$ does not depend on $h$ or $\delta t$. The value of this constant may change at each occurrence.

For the spatial discretization we consider a sequence of quasi-uniform triangulations, and we denote by $h$ the mesh size. The velocity is approximated in $H_{0}^{1}(\Omega)^{d}$ by using continuous 
finite elements composed of piecewise polynomial functions of degree less than or equal to $l$. The corresponding discrete space is denoted by $X_{h}$. Likewise, the pressure is approximated in $L^{2}(\Omega)$ by using continuous finite elements of polynomial degree less than or equal to $l^{\prime}$. The discrete pressure space is denoted by $M_{h}$. From now on, we assume that the discrete spaces $X_{h}$ and $M_{h}$ satisfy the LBB condition

$$
\exists c>0, \sup _{\mathbf{v}_{h} \in X_{h}} \frac{\left(\nabla \cdot \mathbf{v}_{h}, q_{h}\right)}{\left\|\mathbf{v}_{h}\right\|_{1}\left\|q_{h}\right\|_{0}} \geqslant c, \quad \forall q_{h} \in M_{h}
$$

We now introduce elliptic interpolations of $\mathbf{u}(t)$ and $p(t)$ as follows. We define $\mathbf{w}_{h}(t) \in X_{h}$ and $q_{h}(t) \in M_{h}$ as the solution of the following problem:

$$
\begin{aligned}
\left(\nabla \mathbf{w}_{h}(t), \nabla \mathbf{v}_{h}\right)- & \left(q_{h}(t), \nabla \cdot \mathbf{v}_{h}\right)=\left(\nabla \mathbf{u}(t), \nabla \mathbf{v}_{h}\right)-\left(p(t), \nabla \cdot \mathbf{v}_{h}\right), \quad \forall \mathbf{v}_{h} \in X_{h} \\
\left(\nabla \cdot \mathbf{w}_{h}(t), r_{x}\right) & =\left(\nabla \cdot \mathbf{v}(t), r_{x}\right), \quad \forall r_{x} \in M_{h}
\end{aligned}
$$

We recall the following standard results (see e.g. Reference [3] for other details).

\section{Lemma 2.1}

Provided that $\mathbf{u}^{(j)} \in L^{\beta}\left(0, T ; H^{l+1}(\Omega)^{d} \cap V\right), p^{(j)} \in L^{\beta}\left(0, T ; H^{l}(\Omega) \cap L_{0}^{2}(\Omega)\right)$, for $1 \leqslant \beta \leqslant \infty$, there exists $c>0$ such that

$$
\begin{aligned}
& \left\|\mathbf{u}^{(j)}-\mathbf{w}_{h}^{(j)}\right\|_{L^{\beta}\left(0, T ; L^{2}(\Omega)^{d}\right)}+h\left[\left\|\mathbf{u}^{(j)}-\mathbf{w}_{h}^{(j)}\right\|_{L^{\beta}\left(0, T ; H^{1}(\Omega)^{d}\right)}+\left\|p^{(j)}-q_{h}^{(j)}\right\|_{L^{\beta}\left(0, T ; L^{2}(\Omega)\right)}\right] \\
& \quad \leqslant h^{l+1}\left[\left\|\mathbf{u}^{(j)}\right\|_{L^{\beta}\left(0, T ; H^{l+1}(\Omega)^{d}\right)}+\left\|p^{(j)}\right\|_{L^{\beta}\left(0, T ; H^{l}(\Omega)\right)}\right]
\end{aligned}
$$

\section{Lemma 2.2}

Provided that $\mathbf{u}^{(j)} \in L^{\beta}\left(0, T ; H^{2}(\Omega)^{d} \cap H_{0}^{1}(\Omega)^{d}\right), p^{(j)} \in L^{\beta}\left(0, T ; H^{1}(\Omega) \cap L_{0}^{2}(\Omega)\right)$, for $1 \leqslant \beta \leqslant \infty$, there exists $c>0$ such that

$$
\left\|\mathbf{w}_{h}^{(j)}\right\|_{L^{\beta}\left(0, T ; W^{0, \infty}(\Omega)^{d} \cap W^{1,3}(\Omega)^{d}\right)} \leqslant c\left(\left\|\mathbf{u}^{(j)}\right\|_{L^{\beta}\left(0, T ; H^{2}(\Omega)^{d}\right)}+\left\|p^{(j)}\right\|_{L^{\beta}\left(0, T ; H^{1}(\Omega)\right)}\right)
$$

Lemma 2.3

Under the conditions of Lemma 2.2

$$
\left\|\nabla q_{h}^{(j)}\right\|_{L^{\beta}\left(0, T ; L^{2}(\Omega)^{d}\right)} \leqslant c\left(\left\|p^{(j)}\right\|_{L^{\beta}\left(0, T ; H^{1}(\Omega)\right)}+\left\|\mathbf{u}^{(j)}\right\|_{L^{\beta}\left(0, T ; H^{2}(\Omega)\right)}\right)
$$

\subsection{Operator splitting and the method of characteristics}

It is well known (see e.g. Reference [4]) that if we use the method of characteristics to discretize the total time derivative in the Navier-Stokes equations, we obtain the following generalized Stokes problem:

$$
\begin{aligned}
\frac{\mathbf{u}^{n+1}(\mathbf{x})-\underline{\mathbf{u}^{n}}(\mathbf{x})}{\delta t}+\nabla p^{n+1}(\mathbf{x})-\nabla^{2} \mathbf{u}^{n+1}(\mathbf{x}) & =0 \\
\nabla \cdot \mathbf{u}^{n+1}(\mathbf{x}) & =0
\end{aligned}
$$

where the advected velocity $\underline{\mathbf{u}}^{n}(\mathbf{x})$ is defined below (see (12)). As usual, the superscript $n$ denotes the $n$th time level, and we suppose that the time interval $[0, T]$ is discretized 
by a uniform grid $\left\{t^{0}=0, t^{1}, \ldots, t^{N}=T\right\}$ with a grid size $\delta t$. The Reynolds number can be eliminated via a proper rescaling but, of course, the constants in the error estimates will depend implicitly on it. Since in this paper we analyse a first-order projection/characteristic scheme, we present the splitting for the case of the first-order Euler backward time stepping. The same approach can be used to derive second-order schemes.

To define the underlined velocity $\underline{\mathbf{u}}^{n}(\mathbf{x})$, we introduce the so-called characteristic curve $\mathbf{X}_{\mathbf{x}}^{n+1}(s)$ as the solution to the following kinematic problem:

$$
\begin{aligned}
\frac{\mathrm{d} \mathbf{X}_{\mathbf{x}}^{n+1}}{\mathrm{~d} s} & =-\mathbf{u}\left(\mathbf{X}_{\mathbf{x}}^{n+1}(s), t^{n+1}-s\right), \quad s \in(0, \delta t] \\
\mathbf{X}_{\mathbf{x}}^{n+1}(0) & =\mathbf{x}
\end{aligned}
$$

where $\mathbf{u}(\mathbf{y}, t)$ is the Navier-Stokes velocity field, and $\mathbf{x}$ is an arbitrary point within the solution domain where the characteristic curve terminates at time level $t^{n+1}$. We refer to the point $\mathbf{X}_{\mathbf{x}}^{n+1}(\delta t)$ as the foot of the characteristic, and we henceforth denote this field by $\underline{\mathbf{X}}_{\mathbf{x}}^{n+1}$. The convected velocity field $\underline{\mathbf{u}^{n}}(\mathbf{x})$ is defined by

$$
\underline{\mathbf{u}^{n}}(\mathbf{x})=\mathbf{u}^{n}\left(\underline{\mathbf{X}}_{\mathbf{x}}^{n+1}, t^{n}\right)
$$

Since in general $\mathbf{u}\left(\mathbf{X}_{\mathbf{x}}^{n+1}(s), t^{n+1}-s\right)$ cannot be computed exactly, we replace this field by an approximate one $\mathbf{u}^{n *}$ (yet to be defined clearly and assumed to be constant in time). For instance, it is standard to replace the time-dependent velocity field in (11) by $\mathbf{u}\left(\cdot, t^{n}\right)$. The resulting scheme can be shown to be first-order accurate in time. As shown by Boukir et al. [5], if a second-order extrapolation is used, say $2 \mathbf{u}\left(\cdot, t^{n}\right)-\mathbf{u}\left(\cdot, t^{n-1}\right)$, and the time stepping in (10) is modified accordingly, the entire scheme is second-order accurate despite the first-order approximation of the foot of the characteristic.

Henceforth, when an approximate velocity field is used to approximate the characteristic $\mathbf{X}_{\mathbf{x}}^{n+1}(s)$, say $\mathbf{u}^{n *}$, the approximated foot of the characteristic is denoted by $\overline{\mathbf{X}}_{\mathbf{x}}^{n+1}$. For any vector- or scalar-valued field in $\Omega$, say $\eta$, we denote

$$
\underline{\eta}(\mathbf{x})=\eta\left(\underline{\mathbf{X}}_{\mathbf{x}}^{n+1}\right) \quad \text { and } \quad \bar{\eta}(\mathbf{x})=\eta\left(\overline{\mathbf{X}}_{\mathbf{x}}^{n+1}\right)
$$

Let us now recall some stability and approximation results for the method of characteristics. These results are largely due to Douglas and Russell [6] and Süli [7].

\section{Lemma 2.4}

Assume that

$$
\delta t\|\mathbf{u}\|_{L^{\infty}\left(W^{1, \infty}(\Omega)^{d}\right)} \leqslant 1 / 6, \quad \delta t\left\|\mathbf{u}^{n *}\right\|_{1, \infty} \leqslant \varepsilon
$$

where $\varepsilon$ is such that the mapping

$$
\mathbf{x} \rightarrow \overline{\mathbf{X}}_{\mathbf{x}}^{n+1}(s)=\mathbf{x}-s \mathbf{u}^{n *}
$$

has a Jacobian which is greater than or equal to $\frac{1}{2}$ (it has been proved in the above-mentioned studies that such an $\varepsilon$ exists). Then, if the exact solution is smooth enough (see for details Reference [1]). 
(i) for any $\eta \in H^{1}(\Omega)$

$$
\|\bar{\eta}-\underline{\eta}\|_{0,1} \leqslant c \delta t\left(\left\|\mathbf{u}^{n}-\mathbf{u}^{n *}\right\|_{0,2}+\delta t\left\|\mathbf{u}_{t}\right\|_{L^{\infty}\left(L^{2}(\Omega)^{d}\right)}\right)\|\nabla \eta\|_{0,2}
$$

(ii) for any $\eta \in L^{2}(\Omega)$

$$
\|\eta-\underline{\eta}\|_{-1} \leqslant 2 \delta t\|\mathbf{u}\|_{C\left(C^{0,1}(\bar{\Omega})^{d}\right)}\|\eta\|_{0}
$$

\section{ANALYSIS OF THE MODIFIED PROJECTION/CHARACTERISTIC SCHEME}

\subsection{Presentation of the fully discrete scheme}

If we approximate (10) by using the first-order pressure-correction projection method we obtain the following semidiscrete scheme:

$$
\begin{array}{r}
\frac{\tilde{\mathbf{u}}^{n+1}-\mathbf{u}^{n}+\tilde{\mathbf{u}}^{n}-\overline{\tilde{\mathbf{u}}^{n}}}{\delta t}-\nabla^{2} \tilde{\mathbf{u}}^{n+1}=-\nabla p^{n},\left.\quad \tilde{\mathbf{u}}^{n+1}\right|_{\partial \Omega}=0 \\
\frac{\mathbf{u}^{n+1}-\tilde{\mathbf{u}}^{n+1}}{\delta t}=-\nabla\left(p^{n+1}-p^{n}\right), \quad \nabla \cdot \mathbf{u}^{n+1}=0,\left.\quad \mathbf{u}^{n+1} \cdot \mathbf{n}\right|_{\partial \Omega}=0
\end{array}
$$

where $\overline{\tilde{\mathbf{u}}^{n}}(\mathbf{x})$ is computed by using the following definition:

$$
\mathbf{u}^{n *}=\tilde{\mathbf{u}}^{n}
$$

Now we choose the discrete setting for the two velocities and the pressure as follows $\tilde{\mathbf{u}}_{h} \in X_{h}$, $\mathbf{u}_{h} \in Y_{h}=X_{h}+\nabla M_{h}$, and $p_{h} \in M_{h}$. This setting is discussed in details by Guermond and Quartapelle [3]. We denote by $i_{h}$ the injection of $X_{h}$ into $Y_{h}$. It is clear that the adjoint of $i_{h}$, say $i_{h}^{\mathrm{T}}$, is the $L^{2}$ projection of $Y_{h}$ onto $X_{h}$. Using these notations, we can write the fully discrete Galerkin formulation of the projection/characteristic scheme as follows:

Find $\tilde{\mathbf{u}}_{h}^{n+1} \in X_{h}, \mathbf{u}_{h}^{n+1} \in Y_{h}, \quad p_{h}^{n+1} \in M_{h}$ such that

$$
\begin{aligned}
\left(\frac{\tilde{\mathbf{u}}_{h}^{n+1}-i_{h}^{\mathrm{T}} \mathbf{u}_{h}^{n}+\tilde{\mathbf{u}}_{h}^{n}-\overline{\tilde{\mathbf{u}}_{h}^{n}}}{\delta t}, \mathbf{v}_{h}\right) & =\left(p_{h}^{n}, \nabla \cdot \mathbf{v}_{h}\right)-\left(\nabla \tilde{\mathbf{u}}_{h}^{n+1}, \nabla \mathbf{v}_{h}\right), \quad \forall \mathbf{v}_{h} \in X_{h} \\
\left(\frac{\mathbf{u}_{h}^{n+1}-i_{h} \tilde{\mathbf{u}}_{h}^{n+1}}{\delta t}, \mathbf{y}_{h}\right) & =-\left(\nabla\left(p_{h}^{n+1}-p_{h}^{n}\right), \mathbf{y}_{h}\right), \quad \forall \mathbf{y}_{h} \in Y_{h} \\
\left(\mathbf{u}_{h}^{n+1}, \nabla q_{h}\right) & =0, \quad \forall q_{h} \in M_{h}
\end{aligned}
$$

As proved by Guermond and Quartapelle [3] (their Proposition 3.3), the projection part of this scheme (i.e. (21), (22) is equivalent to the following problem:

Find $p_{h}^{n+1} \in M_{h}$ such that

$$
\begin{aligned}
\left(\nabla\left(p_{h}^{n+1}-p_{h}^{n}\right), \nabla q_{h}\right) & =-\frac{1}{\delta t}\left(\nabla \cdot \tilde{\mathbf{u}}_{h}^{n+1}, q_{h}\right), \quad \forall q_{h} \in M_{h} \\
\mathbf{u}_{h}^{n+1} & =\tilde{\mathbf{u}}_{h}^{n+1}-\delta t \nabla\left(p_{h}^{n+1}-p_{h}^{n}\right)
\end{aligned}
$$


Further on, Guermond and Quartapelle [3] and Achdou and Guermond [1] substitute (24) into (20) and thus eliminate the end-of-step velocity $\mathbf{u}_{h}^{n+1}$ from the system. Since (24) is no longer present, the resulting formulation is somewhat more computationally effective than the original one. However, the actually computed velocity field $\tilde{\mathbf{u}}_{h}^{n+1}$ is generally non-solenoidal and one may suspect that its divergence is greater than the divergence of the projected field $i_{h}^{\mathrm{T}} \mathbf{u}_{h}^{n+1}$, though no a priori estimate is known to support this claim. Since characteristic schemes rely on the incompressibility of the convecting field for stability, the lack of control on the divergence of $\tilde{\mathbf{u}}_{h}^{n+1}$ may be a drawback. Therefore, in the present paper we discuss a scheme which does not eliminate the projected velocity but explicitly computes an approximation to it. As the numerical results reported at the end of this paper seem to confirm, in some cases it may be wise to 'waste' resources in this computation to produce a velocity field which allows for the use of larger time steps (although the theoretical limitations on the time step remain of the same order).

Since the projection equation (24) produces a discontinuous (although weakly solenoidal) velocity which cannot be used as the convective field in the Lagrangian step, we simply project it onto $X_{h}$ via the following Galerkin formulation:

Find $i_{h}^{\mathrm{T}} \mathbf{u}_{h} \in X_{h}$ such that

$$
\left(i_{h}^{\mathrm{T}} \mathbf{u}_{h}^{n+1}, \mathbf{v}_{h}\right)=\left(\tilde{\mathbf{u}}_{h}^{n+1}, \mathbf{v}_{h}\right)-\delta t\left(\nabla\left(p_{h}^{n+1}-p_{h}^{n}\right), \mathbf{v}_{h}\right), \quad \forall \mathbf{v}_{h} \in X_{h}
$$

$i_{h}^{\mathrm{T}}$ being a projection, we clearly have

$$
\left\|i_{h}^{\mathrm{T}} \mathbf{u}_{h}^{n}\right\|_{0} \leqslant\left\|\mathbf{u}_{h}^{n}\right\|_{0}
$$

A small extra computational effort is necessary for inverting the mass matrix that arises from (25). However, since the mass matrix is well conditioned, a properly preconditioned iterative method (using for example its lumped version) can invert it very effectively. In fact, this scheme is close in spirit to the one of Gresho and Chan [8], part II. From now on we use the following definition for computing the foot of characteristics:

$$
\mathbf{u}^{n *}=i_{h}^{\mathrm{T}} \mathbf{u}_{h}^{n}
$$

Consequently, we modify our projection scheme as follows:

Find $\tilde{\mathbf{u}}_{h}^{n+1} \in X_{h}, \mathbf{u}_{h}^{n+1} \in Y_{h}, \quad$ and $\quad p_{h}^{n+1} \in M_{h}$ such that

$$
\begin{aligned}
\left(\frac{\tilde{\mathbf{u}}_{h}^{n+1}-\overline{i_{h}^{\mathrm{T}} \mathbf{u}_{h}^{n}}}{\delta t}, \mathbf{v}_{h}\right) & =\left(p_{h}^{n}, \nabla \cdot \mathbf{v}_{h}\right)-\left(\nabla \tilde{\mathbf{u}}_{h}^{n+1}, \nabla \mathbf{v}_{h}\right), \quad \forall \mathbf{v}_{h} \in X_{h} \\
\left(\frac{\mathbf{u}_{h}^{n+1}-i_{h} \tilde{\mathbf{u}}_{h}^{n+1}}{\delta t}, \mathbf{y}_{h}\right) & =-\left(\nabla\left(p_{h}^{n+1}-p_{h}^{n}\right), \mathbf{y}_{h}\right), \quad \forall \mathbf{y}_{h} \in Y_{h} \\
\left(\mathbf{u}_{h}^{n+1}, \nabla q_{h}\right) & =0, \quad \forall q_{h} \in M_{h}
\end{aligned}
$$

The convergence analysis of this scheme is reported in the next section.

\subsection{Convergence analysis}

The main result of this paper is the following theorem. 
Theorem 3.1

If the exact solution $\mathbf{u}, p$, and the initial data are smooth enough, (see for details Reference [1]), there exist $c_{e}, c_{s}>0$ and $h_{s}$ such that for all $\left.\left.h \in\right] 0, h_{s}\right]$, and $\delta t \leqslant c_{s} h^{d / 3}$

$$
\begin{aligned}
\left\|\mathbf{u}-\mathbf{u}_{h}\right\|_{\ell^{\infty}\left(L^{2}(\Omega)^{d}\right)}+\left\|\mathbf{u}-\tilde{\mathbf{u}}_{h}\right\|_{\ell^{\infty}\left(L^{2}(\Omega)^{d}\right)} & \leqslant c_{e}\left(h^{l+1}+\delta t\right) \\
\left\|\mathbf{u}-\tilde{\mathbf{u}}_{h}\right\|_{\ell^{2}\left(H^{1}(\Omega)^{d}\right)} & \leqslant c_{e}\left(h^{l}+\delta t\right)
\end{aligned}
$$

Proof

Let us first introduce the following notations for the approximation errors:

$$
\tilde{\mathbf{e}}_{h}^{n}=\mathbf{w}_{h}^{n}-\tilde{\mathbf{u}}_{h}^{n}, \quad \mathbf{e}_{h}^{n}=\mathbf{w}_{h}^{n}-\mathbf{u}_{h}^{n}, \quad \varepsilon_{h}^{n}=q_{h}^{n}-p_{h}^{n}
$$

and the interpolation error

$$
\tilde{\mathbf{e}}_{I}^{n}=\mathbf{w}_{h}^{n}-\mathbf{u}^{n}
$$

Similarly to References $[1,5,7]$ the proof proceeds by induction. Let $\varepsilon>0$ be such that the hypotheses of Lemma 2.4 hold. Under proper initialization assumptions we can show (see Reference [1]) that

$$
\begin{gathered}
\left\|\mathbf{u}_{0}-\mathbf{u}_{h}^{0}\right\|_{0}+\left\|\mathbf{u}_{0}-\tilde{\mathbf{u}}_{h}^{0}\right\|_{0}+h\left\|\mathbf{u}_{0}-\mathbf{u}_{h}^{0}\right\|_{1} \leqslant c h^{l+1} \\
\delta t\left\|i_{h}^{\mathrm{T}} \mathbf{u}_{h}^{0}\right\|_{1, \infty} \leqslant \varepsilon
\end{gathered}
$$

Then, the induction hypothesis is that there exist $c_{s}>0, c_{e}>0$ and $h_{s}>0$ such that at time step $t^{m}, 0 \leqslant m \leqslant T / \delta t$ and for all $h<h_{s}$ and $\delta t \leqslant c_{s} h^{d / 3}$ we have

$$
\begin{gathered}
\left\|\mathbf{u}-\mathbf{u}_{h}\right\|_{\ell^{\infty}\left(0, t^{m} ; L^{2}(\Omega)^{d}\right)}+\left\|\mathbf{u}-\tilde{\mathbf{u}}_{h}\right\|_{\ell^{\infty}\left(0, t^{m} ; L^{2}(\Omega)^{d}\right)} \\
\| c_{e}\left(h^{l+1}+\delta t\right) \\
\left\|\mathbf{u}-\tilde{\mathbf{u}}_{h}\right\|_{\ell^{2}\left(0, t^{m} ; H^{1}(\Omega)^{d}\right)} \leqslant c_{e}\left(h^{l}+\delta t\right) \\
\delta t\left\|i_{h}^{\mathrm{T}} \mathbf{u}_{h}^{m}\right\|_{1, \infty} \leqslant \varepsilon
\end{gathered}
$$

Now we shall prove that these estimates hold at the next time level $m+1$.

Given the particular choice we have made for the interpolating functions $\mathbf{w}_{h}$ and $q_{h}$, the exact solution of the boundary-value problem (1-3) satisfies

$$
\left(\frac{\mathbf{w}_{h}^{n+1}-\overline{i_{h}^{\mathrm{T}} \mathbf{w}_{h}^{n}}}{\delta t}, \mathbf{v}_{h}\right)+\left(\nabla \mathbf{w}_{h}^{n+1}, \nabla \mathbf{v}_{h}\right)-\left(q_{h}^{n+1}, \nabla \cdot \mathbf{v}_{h}\right)=\left(\frac{\mathbf{w}_{h}^{n+1}-\overline{\mathbf{w}_{h}^{n}}}{\delta t}, \mathbf{v}_{h}\right)-\left(\frac{\mathrm{D} \mathbf{u}}{\mathrm{D} t}\left(\mathbf{x}, t^{n+1}\right), \mathbf{v}_{h}\right)
$$

Note that we have used $i_{h}^{\mathrm{T}} \mathbf{w}_{h}=\mathbf{w}_{h}$. Subtracting this equation from (28), we obtain

$$
\begin{gathered}
\left(\frac{\tilde{\mathbf{e}}_{h}^{n+1}-\overline{i_{h}^{\mathrm{T}} \mathbf{e}_{h}^{n}}}{\delta t}, \mathbf{v}_{h}\right)+\left(\nabla \tilde{\mathbf{e}}_{h}^{n+1}, \nabla \mathbf{v}_{h}\right)-\left(\psi_{h}^{n}, \nabla \cdot \mathbf{v}_{h}\right) \\
=\left(\frac{\mathbf{w}_{h}^{n+1}-\overline{\mathbf{w}_{h}^{n}}}{\delta t}, \mathbf{v}_{h}\right)-\left(\frac{\mathrm{D} \mathbf{u}}{\mathrm{D} t}\left(\mathbf{x}, t^{n+1}\right), \mathbf{v}_{h}\right)
\end{gathered}
$$


After rearranging the terms, we have

$$
\begin{aligned}
( & \left.\frac{\tilde{\mathbf{e}}_{h}^{n+1}-i_{h}^{\mathrm{T}} \mathbf{e}_{h}^{n}}{\delta t}, \mathbf{v}_{h}\right)+\left(\nabla \tilde{\mathbf{e}}_{h}^{n+1}, \nabla \mathbf{v}_{h}\right)-\left(\psi_{h}^{n}, \nabla \cdot \mathbf{v}_{h}\right) \\
= & \left(\frac{\mathbf{w}_{h}^{n+1}-\overline{\mathbf{w}_{h}^{n}}}{\delta t}, \mathbf{v}_{h}\right)+\left(\frac{\overline{i_{h}^{\mathrm{T}} \mathbf{e}_{h}^{n}}-i_{h}^{\mathrm{T}} \mathbf{e}_{h}^{n}}{\delta t}, \mathbf{v}_{h}\right) \\
& -\left(\frac{\mathbf{u}^{n+1}-\underline{\mathbf{u}^{n}}}{\delta t}, \mathbf{v}_{h}\right)-\frac{1}{\delta t}\left(\int_{t^{n}}^{t^{n+1}}\left(t-t^{n}\right) \frac{\mathrm{D}^{2} \mathbf{u}}{\mathrm{D} t^{2}}(\mathbf{x}(t), t) \mathrm{d} t, \mathbf{v}_{h}\right) \\
= & -\frac{1}{\delta t}\left(\int_{t^{n}}^{t^{n+1}}\left(t-t^{n}\right) \frac{\mathrm{D}^{2} \mathbf{u}}{\mathrm{D} t^{2}}(\mathbf{x}(t), t) \mathrm{d} t, \mathbf{v}_{h}\right)+\left(\frac{\underline{\mathbf{u}}^{n}-\overline{\mathbf{u}^{n}}}{\delta t}, \mathbf{v}_{h}\right) \\
& +\left(\frac{\mathbf{e}_{I}^{n+1}-\overline{\mathbf{e}_{I}^{n}}}{\delta t}, \mathbf{v}_{h}\right)+\left(\frac{\overline{i_{h}^{\mathrm{T}} \mathbf{e}_{h}^{n}}-i_{h}^{\mathrm{T}} \mathbf{e}_{h}^{n}}{\delta t}, \mathbf{v}_{h}\right)=\sum_{i=1}^{4} R_{i}\left(\mathbf{v}_{h}\right)
\end{aligned}
$$

where we have set

$$
\begin{aligned}
& R_{1}\left(\mathbf{v}_{h}\right)=-\frac{1}{\delta t}\left(\int_{t^{n}}^{t^{n+1}}\left(t-t^{n}\right) \frac{\mathrm{D}^{2} \mathbf{u}}{\mathrm{D} t^{2}}(\mathbf{x}(t), t) \mathrm{d} t, \mathbf{v}_{h}\right), R_{2}\left(\mathbf{v}_{h}\right)=\left(\frac{\mathbf{u}^{n}-\overline{\mathbf{u}^{n}}}{\delta t}, \mathbf{v}_{h}\right) \\
& R_{3}\left(\mathbf{v}_{h}\right)=\left(\frac{\mathbf{e}_{I}^{n+1}-\overline{\mathbf{e}_{I}^{n}}}{\delta t}, \mathbf{v}_{h}\right), R_{4}\left(\mathbf{v}_{h}\right)=\left(\frac{\overline{i_{h}^{\mathrm{T}} \mathbf{e}_{h}^{n}}-i_{h}^{\mathrm{T}} \mathbf{e}_{h}^{n}}{\delta t}, \mathbf{v}_{h}\right)
\end{aligned}
$$

and $\psi_{h}^{n}=q_{h}^{n+1}-q_{h}^{n}+\varepsilon_{h}^{n}$. Choosing $\mathbf{v}_{h}=2 \delta t \tilde{\mathbf{e}}_{h}^{n+1}$ and using the equality $2(a, a-b)=\|a\|^{2}+$ $\|a-b\|^{2}-\|b\|^{2}$ together with the Poincaré-Friedrichs inequality (the constant $\alpha$ is precisely the ellipticity constant of the bilinear form $(\nabla \cdot, \nabla \cdot))$ we obtain

$$
\left\|\tilde{\mathbf{e}}_{h}^{n+1}\right\|_{0}^{2}+\left\|\tilde{\mathbf{e}}_{h}^{n+1}-i_{h}^{\mathrm{T}} \mathbf{e}_{h}^{n}\right\|_{0}^{2}-\left\|i_{h}^{\mathrm{T}} \mathbf{e}_{h}^{n}\right\|_{0}^{2}+2 \alpha \delta t\left\|\tilde{\mathbf{e}}_{h}^{n+1}\right\|_{1}^{2}+2 \delta t\left(\tilde{\mathbf{e}}_{h}^{n+1}, \nabla \psi_{h}^{n}\right) \leqslant 2 \delta t \sum_{i=1}^{4}\left|R_{i}\left(\tilde{\mathbf{e}}_{h}^{n+1}\right)\right|
$$

From (24) we easily obtain

$$
\frac{\mathbf{e}_{h}^{n+1}-i_{h} \tilde{\mathbf{e}}_{h}^{n+1}}{\delta t}+\nabla\left(\varepsilon_{h}^{n+1}-\psi_{h}^{n}\right)=0
$$

and from (22)

$$
\left(\mathbf{e}_{h}^{n+1}, \nabla q_{h}\right)=0
$$

Multiplying (45) by $2 \delta t^{2} \nabla \psi_{h}^{n}$ and using Lemma 2.3 we infer

$$
\begin{aligned}
-2 \delta t\left(\tilde{\mathbf{e}}_{h}^{n+1}, \nabla \psi_{h}^{n}\right)+\delta t^{2}\left\|\nabla \varepsilon_{h}^{n+1}\right\|_{0}^{2}-\left\|\mathbf{e}_{h}^{n+1}-i_{h} \tilde{\mathbf{e}}_{h}^{n+1}\right\|_{0}^{2} & =\delta t^{2}\left\|\nabla \psi_{h}^{n}\right\|_{0}^{2} \\
& \leqslant \delta t^{2}(1+\delta t)\left\|\nabla \varepsilon_{h}^{n}\right\|_{0}^{2}+c \delta t^{3}
\end{aligned}
$$


Multiplying the same equation by $2 \delta t \mathbf{e}_{h}^{n+1}$ we have

$$
\left\|\mathbf{e}_{h}^{n+1}\right\|_{0}^{2}+\left\|\mathbf{e}_{h}^{n+1}-i_{h} \tilde{\mathbf{e}}_{h}^{n+1}\right\|_{0}^{2}-\left\|\tilde{\mathbf{e}}_{h}^{n+1}\right\|_{0}^{2}=0
$$

Summing (44), (47), and (48) and taking into account (26) we obtain

$$
\begin{aligned}
& \left\|\mathbf{e}_{h}^{n+1}\right\|_{0}^{2}+\left\|\tilde{\mathbf{e}}_{h}^{n+1}-i_{h}^{\mathrm{T}} \mathbf{e}_{h}^{n}\right\|_{0}^{2}+2 \alpha \delta t\left\|\tilde{\mathbf{e}}_{h}^{n+1}\right\|_{1}^{2}+\delta t^{2}\left\|\nabla \varepsilon_{h}^{n+1}\right\|_{0}^{2} \\
& \quad \leqslant 2 \delta t \sum_{i=1}^{4}\left|R_{i}\left(\tilde{\mathbf{e}}_{h}^{n+1}\right)\right|+\left\|\mathbf{e}_{h}^{n}\right\|_{0}^{2}+\delta t^{2}(1+\delta t)\left\|\nabla \varepsilon_{h}^{n}\right\|_{0}^{2}+c \delta t^{3}
\end{aligned}
$$

Estimates for $R_{i}\left(\tilde{\mathbf{e}}_{h}^{n+1}\right), i=1,2,3,4$ can be obtained as follows:

(i) Using the classical inequality $(a, b) \leqslant \gamma\|a\|^{2}+1 / 4 \gamma\|b\|^{2}, \forall \gamma>0$, we deduce.

$$
2 \delta t\left|R_{1}^{n+1}\left(\tilde{\mathbf{e}}_{h}^{n+1}\right)\right| \leqslant \gamma \delta t\left\|\tilde{\mathbf{e}}_{h}^{n+1}\right\|_{1}^{2}+c \delta t^{3}
$$

where $\gamma$ is a constant to be fixed later.

(ii) Concerning the second non-linear residual we have

$$
\begin{aligned}
2 \delta t\left|R_{2}^{n+1}\left(\tilde{\mathbf{e}}_{h}^{n+1}\right)\right| & \leqslant \delta t\left(\gamma\left\|\tilde{\mathbf{e}}_{h}^{n+1}\right\|_{0}^{2}+\frac{1}{\gamma}\left\|\frac{\mathbf{u}^{n}-\overline{\mathbf{u}^{n}}}{\delta t}\right\|_{0}^{2}\right) \\
& \leqslant \delta t\left(\gamma\left\|\tilde{\mathbf{e}}_{h}^{n+1}\right\|_{0}^{2}+c\left\|\mathbf{u}^{n}\right\|_{1, \infty}^{2}\left\|\mathbf{u}^{n}-i_{h}^{\mathrm{T}} \mathbf{u}_{h}^{n}\right\|_{0}^{2}\right) \\
& \leqslant \delta t\left(\gamma\left\|\tilde{\mathbf{e}}_{h}^{n+1}\right\|_{0}^{2}+c\left(\left\|\mathbf{u}^{n}-\mathbf{w}_{h}^{n}\right\|_{0}^{2}+\left\|\mathbf{w}_{h}^{n}-i_{h}^{\mathrm{T}} \mathbf{u}_{h}^{n}\right\|_{0}^{2}\right)\right) \\
& \leqslant \delta t\left(\gamma\left\|\tilde{\mathbf{e}}_{h}^{n+1}\right\|_{1}^{2}+c^{\prime} h^{2(l+1)}+c\left\|\mathbf{e}_{h}^{n}\right\|_{0}^{2}\right)
\end{aligned}
$$

In the last estimate we used the interpolation property of $\mathbf{w}_{h}^{n}$ given by Lemma 2.1 .

(iii) For the third non-linear residual we proceeds as follows:

$$
\begin{aligned}
2 \delta t\left|R_{3}^{n+1}\left(\tilde{\mathbf{e}}_{h}^{n+1}\right)\right|= & 2\left|\left(\mathbf{e}_{I}^{n+1}-\overline{\mathbf{e}_{I}^{n}}, \tilde{\mathbf{e}}_{h}^{n+1}\right)\right| \\
\leqslant & 2\left(\left|\left(\tilde{\mathbf{e}}_{h}^{n+1}, \mathbf{e}_{I}^{n+1}-\mathbf{e}_{I}^{n}\right)\right|+\left|\left(\tilde{\mathbf{e}}_{h}^{n+1}, \mathbf{e}_{I}^{n}-\underline{\mathbf{e}_{I}^{n}}\right)\right|+\left|\left(\tilde{\mathbf{e}}_{h}^{n+1}, \underline{\mathbf{e}_{I}^{n}}-\overline{\mathbf{e}_{I}^{n}}\right)\right|\right) \\
\leqslant & 2\left(\left\|\tilde{\mathbf{e}}_{h}^{n+1}\right\|_{0}\left\|\mathbf{e}_{I}^{n+1}-\mathbf{e}_{I}^{n}\right\|_{0}+\left\|\tilde{\mathbf{e}}_{h}^{n+1}\right\|_{1}\left\|\mathbf{e}_{I}^{n}-\underline{\mathbf{e}_{I}^{n}}\right\|_{-1}\right. \\
& \left.+\left\|\tilde{\mathbf{e}}_{h}^{n+1}\right\|_{0, \infty}\left\|\underline{\mathbf{e}_{I}^{n}}-\overline{\mathbf{e}_{I}^{n}}\right\|_{0,1}\right)
\end{aligned}
$$

Each of the three terms in the right-hand side of the last inequality can be estimated as follows:

$$
\begin{aligned}
2\left\|\tilde{\mathbf{e}}_{h}^{n+1}\right\|_{0}\left\|\mathbf{e}_{I}^{n+1}-\mathbf{e}_{I}^{n}\right\|_{0} & \leqslant \gamma \delta t\left\|\tilde{\mathbf{e}}_{h}^{n+1}\right\|_{1}^{2}+c\left\|\partial_{t} \mathbf{e}_{I}\right\|_{L^{2}\left(t^{n}, t^{n+1} ; L^{2}(\Omega)^{d}\right)}^{2} \\
& \leqslant \gamma \delta t\left\|\tilde{\mathbf{e}}_{h}^{n+1}\right\|_{1}^{2}+c \delta t h^{2(l+1)}
\end{aligned}
$$


Using Lemma 2.4 and the interpolation properties stated in Lemma 2.1 we have

$$
\begin{aligned}
\left\|\tilde{\mathbf{e}}_{h}^{n+1}\right\|_{1}\left\|\mathbf{e}_{I}^{n}-\underline{\mathbf{e}}_{I}^{n}\right\|_{-1} & \leqslant 2 \delta t\left\|\tilde{\mathbf{e}}_{h}^{n+1}\right\|_{1}\|\mathbf{u}\|_{C\left(C^{0,1}(\bar{\Omega})^{d}\right)}\left\|\mathbf{e}_{I}^{n}\right\|_{0} \\
& \leqslant \gamma \delta t\left\|\tilde{\mathbf{e}}_{h}^{n+1}\right\|_{1}^{2}+c \delta t h^{2(l+1)}
\end{aligned}
$$

Upon using an inverse inequality (see Reference [9, p. 140]) we obtain

$$
\begin{aligned}
2\left\|\tilde{\mathbf{e}}_{h}^{n+1}\right\|_{0, \infty}\left\|\underline{\mathbf{e}_{I}^{n}}-\overline{\mathbf{e}_{I}^{n}}\right\|_{0,1} & \leqslant c \delta t D(h)\left\|\tilde{\mathbf{e}}_{h}^{n+1}\right\|_{1}\left(\left\|\mathbf{u}_{n}-i_{h}^{\mathrm{T}} \mathbf{u}_{h}^{n}\right\|_{0,2}+\delta t\left\|\partial_{t} \mathbf{u}\right\|_{L^{\infty}\left(L^{2}(\Omega)^{d}\right)}\right)\left\|\nabla \mathbf{e}_{I}^{n}\right\|_{0,2} \\
& \leqslant c \delta t D(h)\left\|\mathbf{e}_{I}^{n}\right\|_{1}\left(\left\|\mathbf{e}_{I}^{n}\right\|_{0}+\left\|i_{h}^{\mathrm{T}} \mathbf{e}_{h}^{n}\right\|_{0}+c \delta t\right)\left\|\tilde{\mathbf{e}}_{h}^{n+1}\right\|_{1}
\end{aligned}
$$

Here $D(h)$ comes from the inverse estimate (of course, we assume that the grid satisfies the inverse assumption, see Reference [9])

$$
D(h)= \begin{cases}(1+|\log h|)^{1 / 2} & \text { in 2D } \\ h^{-1 / 2} & \text { in 3D }\end{cases}
$$

The interpolation properties of $\mathbf{w}_{h}$ now clearly imply that if $h_{s}$ is small enough then $c D(h)\left\|\mathbf{e}_{I}^{n}\right\|_{1}$ $\leqslant 1$. Therefore,

$$
2\left\|\tilde{\mathbf{e}}_{h}^{n+1}\right\|_{0, \infty}\left\|\underline{\mathbf{e}_{I}^{n}}-\overline{\mathbf{e}_{I}^{n}}\right\|_{0,1} \leqslant \gamma \delta t\left\|\tilde{\mathbf{e}}_{h}^{n+1}\right\|_{1}^{2}+\delta t\left\|\mathbf{e}_{h}^{n}\right\|_{0}^{2}+c \delta t\left(\delta t^{2}+h^{l+1}\right)^{2}
$$

Finally we obtain the following bound:

$$
2 \delta t\left|R_{3}^{n+1}\left(\tilde{\mathbf{e}}_{h}^{n+1}\right)\right| \leqslant 3 \gamma \delta t\left\|\tilde{\mathbf{e}}_{h}^{n+1}\right\|_{1}^{2}+\delta t\left\|\mathbf{e}_{h}^{n}\right\|_{0}^{2}+c \delta t\left(\delta t^{2}+h^{l+1}\right)^{2}
$$

(iv) We treat the fourth non-linear residual as follows:

$$
\left.\left.2 \delta t\left|R_{4}^{n+1}\left(\tilde{\mathbf{e}}_{h}^{n+1}\right)\right| \leqslant 2\left(\| \underline{i_{h}^{\mathrm{T}} \mathbf{e}_{h}^{n}}-i_{h}^{\mathrm{T}} \mathbf{e}_{h}^{n}\right)\left\|_{-1}\right\| \tilde{\mathbf{e}}_{h}^{n+1}\left\|_{1}+\right\| \overline{i_{h}^{\mathrm{T}} \mathbf{e}_{h}^{n}}-\underline{i_{h}^{\mathrm{T}} \mathbf{e}_{h}^{n}}\right)\left\|_{0,1}\right\| \tilde{\mathbf{e}}_{h}^{n+1} \|_{0, \infty}\right)
$$

Using Lemma 2.1 and Young's inequality, we obtain the following estimates for the terms in the right-hand side:

$$
\begin{gathered}
2\left\|\underline{i_{h}^{\mathrm{T}} \mathbf{e}_{h}^{n}}-i_{h}^{\mathrm{T}} \mathbf{e}_{h}^{n}\right\|_{-1}\left\|\tilde{\mathbf{e}}_{h}^{n+1}\right\|_{1} \leqslant c \delta t\left\|\mathbf{e}_{h}^{n}\right\|_{0}\left\|\tilde{\mathbf{e}}_{h}^{n+1}\right\|_{1} \leqslant \delta t\left(c\left\|\mathbf{e}_{h}^{n}\right\|_{0}^{2}+\gamma\left\|\tilde{\mathbf{e}}_{h}^{n+1}\right\|_{1}^{2}\right) \\
2\left\|\overline{i_{h}^{\mathrm{T}} \mathbf{e}_{h}^{n}}-\underline{i_{h}^{\mathrm{T}} \mathbf{e}_{h}^{n}}\right\|_{0,1}\left\|\tilde{\mathbf{e}}_{h}^{n+1}\right\|_{0, \infty} \leqslant c \delta t\left(\left\|\mathbf{e}_{I}^{n}\right\|_{0}+\left\|i_{h}^{\mathrm{T}} \mathbf{e}_{h}^{n}\right\|_{0}+\delta t\left\|\partial_{t} \mathbf{u}\right\|_{L^{\infty}\left(L^{2}(\Omega)^{d}\right)}\right)\left\|i_{h}^{\mathrm{T}} \mathbf{e}_{h}^{n}\right\|_{1} D(h)\left\|\tilde{\mathbf{e}}_{h}^{n+1}\right\|_{1}
\end{gathered}
$$

Taking into account the induction hypothesis and the interpolation error estimate we obtain

$$
2\left\|i_{h}^{\mathrm{T}}\left(\overline{\mathbf{e}_{h}^{n}}-\underline{\mathbf{e}_{h}^{n}}\right)\right\|_{0,1}\left\|\tilde{\mathbf{e}}_{h}^{n+1}\right\|_{0, \infty} \leqslant c \delta t D(h)\left(h^{l+1}+\delta t\right)\left\|\tilde{\mathbf{e}}_{h}^{n+1}\right\|_{1}\left\|i_{h}^{\mathrm{T}} \mathbf{e}_{h}^{n}\right\|_{1}
$$

It is at this point that the error analysis really departs from that in Reference [1]. Here we have not yet an a priori control on $\left\|i_{h}^{\mathrm{T}} \mathbf{e}_{h}^{n}\right\|_{1}^{2}$. To obtain such a control we proceed as follows:

$$
\begin{aligned}
\left\|i_{h}^{\mathrm{T}} \mathbf{e}_{h}^{n}\right\|_{1} & \leqslant\left\|\tilde{\mathbf{e}}_{h}^{n+1}-i_{h}^{\mathrm{T}} \mathbf{e}_{h}^{n}\right\|_{1}+\left\|\tilde{\mathbf{e}}_{h}^{n+1}\right\|_{1} \\
& \leqslant c h^{-1}\left\|\tilde{\mathbf{e}}_{h}^{n+1}-i_{h}^{\mathrm{T}} \mathbf{e}_{h}^{n}\right\|_{0}+\left\|\tilde{\mathbf{e}}_{h}^{n+1}\right\|_{1}
\end{aligned}
$$


Then, we have

$$
\begin{aligned}
2\left\|i_{h}^{\mathrm{T}}\left(\overline{\mathbf{e}_{h}^{n}}-\underline{\mathbf{e}_{h}^{n}}\right)\right\|_{0,1}\left\|\tilde{\mathbf{e}}_{h}^{n+1}\right\|_{0, \infty} \leqslant & \delta t\left(\gamma+D(h)\left(h^{l+1}+\delta t\right)\right)\left\|\tilde{\mathbf{e}}_{h}^{n+1}\right\|_{1}^{2} \\
& +c \delta t\left(h^{-1} D(h)\left(h^{l+1}+\delta t\right)\right)^{2}\left\|\tilde{\mathbf{e}}_{h}^{n+1}-i_{h}^{\mathrm{T}} \mathbf{e}_{h}^{n}\right\|_{0}^{2}
\end{aligned}
$$

It is clear that if $h$ is small enough and $\delta t \leqslant c_{s} h^{d / 3}$ with $c_{s}$ small enough, then $D(h)\left(h^{l+1}+\delta t\right) \leqslant \gamma$ and $c \delta t\left(h^{-1} D(h)\left(h^{l+1}+\delta t\right)\right)^{2} \leqslant 1 / 2$. As a result,

$$
2 \delta t\left|R_{4}^{n+1}\left(\tilde{\mathbf{e}}_{h}^{n+1}\right)\right| \leqslant 3 \gamma \delta t\left\|\tilde{\mathbf{e}}_{h}^{n+1}\right\|_{1}^{2}+c \delta t\left\|\mathbf{e}_{h}^{n}\right\|_{0}^{2}+\frac{1}{2}\left\|\tilde{\mathbf{e}}_{h}^{n+1}-i_{h}^{\mathrm{T}} \mathbf{e}_{h}^{n}\right\|_{0}^{2}
$$

Finally, substituting the estimates for $R_{i}^{n+1}$ in (49) we obtain

$$
\begin{aligned}
& \left\|\mathbf{e}_{h}^{n+1}\right\|_{0}^{2}+\delta t^{2}\left\|\nabla \varepsilon_{h}^{n+1}\right\|_{0}^{2}+\frac{1}{2}\left\|\tilde{\mathbf{e}}_{h}^{n+1}-i_{h}^{\mathrm{T}} \mathbf{e}_{h}^{n}\right\|_{0}^{2}+(2 \alpha-8 \gamma) \delta t\left\|\tilde{\mathbf{e}}_{h}^{n+1}\right\|_{1}^{2} \\
& \quad \leqslant(1+c \delta t)\left(\left\|\mathbf{e}_{h}^{n}\right\|_{0}^{2}+\delta t^{2}\left\|\nabla \varepsilon_{h}^{n}\right\|_{0}^{2}\right)+c^{\prime} \delta t\left(\delta t+h^{l+1}\right)^{2}
\end{aligned}
$$

We now choose $\gamma=\alpha / 8$. Using the discrete Gronwall lemma and the initialization hypothesis we obtain

$$
\left\|\mathbf{e}_{h}^{m+1}\right\|_{0}^{2}+\delta t^{2}\left\|\nabla \varepsilon_{h}^{m+1}\right\|_{0}^{2}+\sum_{n=1}^{m}\left\|\tilde{\mathbf{e}}_{h}^{n+1}-i_{h}^{\mathrm{T}} \mathbf{e}_{h}^{n}\right\|_{0}^{2}+\alpha \delta t \sum_{n=1}^{m}\left\|\tilde{\mathbf{e}}_{h}^{n+1}\right\|_{1}^{2} \leqslant c\left(\delta t+h^{l+1}\right)^{2}
$$

Note that in this estimate, the constant $c$ depends only on the data. Provided $T$ is bounded, $c$ does not depend on $m$, $\delta t$, or $h$. From this estimate, the induction hypothesis follows immediately taking into account that

$$
\mathbf{u}^{n}-\mathbf{u}_{h}^{n}=\mathbf{e}_{h}^{n}-\mathbf{e}_{I}^{n}, \quad \mathbf{u}^{n}-\tilde{\mathbf{u}}_{h}^{n}=\tilde{\mathbf{e}}_{h}^{n}-\mathbf{e}_{I}^{n}
$$

Now it remains to prove the stability hypothesis of Lemma 2.4

$$
\left\|i_{h}^{\mathrm{T}} \mathbf{u}_{h}^{m+1}\right\|_{1, \infty} \delta t \leqslant \varepsilon
$$

At this point we cannot use the same argument as in Reference [1] since we do not control the $H^{1}$ norm of $i_{h}^{\mathrm{T}} \mathbf{u}_{h}^{m+1}$; the only a priori control we have up to now is the one on the $L^{2}$ norm of this quantity. To bridge the gap we use a new result stated below in Lemma 3.2. Owing to this lemma we have $\left\|i_{h}^{\mathrm{T}} \mathbf{u}_{h}^{m+1}\right\|_{1} \leqslant c\left\|\tilde{\mathbf{u}}_{h}^{m+1}\right\|_{1}$. This estimate yields $\left\|i_{h}^{\mathrm{T}} \mathbf{u}_{h}^{m+1}\right\|_{1} \leqslant c$, for $\left\|\tilde{\mathbf{u}}_{h}^{m+1}\right\|_{1}$ is bounded, thanks to the a priori error estimates already at hand and an inverse inequality in time. Then the rest of the proof uses inverse inequalities in exactly the same fashion as done by Achdou and Guermond [1] and Boukir et al. [5]. This completes the proof of the theorem.

\section{Lemma 3.1}

Let $\widetilde{i_{h}^{\mathrm{T}}}: L^{2}(\Omega)^{d} \rightarrow X_{h}$ be the $L^{2}$ projection onto $X_{h}$ (note that $\widetilde{i_{h}^{\mathrm{T}}}$ is an extension of $i_{h}^{\mathrm{T}}$ ). Then, there is $c>0$, independent of $h$, such that

$$
\left\|\widetilde{i_{h}^{\mathrm{T}} \mathbf{v}}\right\|_{1} \leqslant c\|\mathbf{v}\|_{1}, \quad \forall \mathbf{v} \in H^{1}(\Omega)^{d}
$$


Proof

Let $\mathscr{I}_{h}$ an interpolation operator on $X_{h}$ stable in $H^{1}(\Omega)^{d}$ and invariant on $X_{h}$, i.e., e.g. the elliptic interpolation. Then we have

$$
\begin{aligned}
\left\|\widetilde{i_{h}^{\mathrm{T}}} \mathbf{v}\right\|_{1} & \leqslant\left\|\widetilde{i_{h}^{\mathrm{T}}} \mathbf{v}-\mathscr{I}_{h} \mathbf{v}\right\|_{1}+\left\|\mathscr{I}_{h} \mathbf{v}\right\|_{1} \\
& \leqslant\left\|\tilde{i}_{h}^{\mathrm{T}}\left(\mathbf{v}-\mathscr{I}_{h} \mathbf{v}\right)\right\|_{1}+c^{\prime}\|\mathbf{v}\|_{1} \\
& \leqslant c h^{-1}\left\|\widetilde{i_{h}^{\mathrm{T}}}\left(\mathbf{v}-\mathscr{I}_{h} \mathbf{v}\right)\right\|_{0}+c^{\prime}\|\mathbf{v}\|_{1} \\
& \leqslant c h^{-1}\left\|\mathbf{v}-\mathscr{I}_{h} \mathbf{v}\right\|_{0}+c^{\prime}\|\mathbf{v}\|_{1} \\
& \leqslant c\|\mathbf{v}\|_{1}
\end{aligned}
$$

The proof is complete.

Lemma 3.2

Let $\mathbf{u} \in H_{0}^{1}(\Omega)^{d}$. Define $\phi_{h} \in M_{h}$ s.t.

$$
\left(\nabla \phi_{h}, \nabla q_{h}\right)=\left(\mathbf{u}, \nabla q_{h}\right), \quad \forall q_{h} \in M_{h}
$$

and set $\mathbf{u}_{h}=\mathbf{u}-\nabla \phi_{h}$. Then, provided $\Omega$ is smooth enough, there is a constant $c$ independent of $h$ such that $\left\|i_{h}^{\mathrm{T}} \mathbf{u}_{h}\right\|_{1} \leqslant c\|\mathbf{u}\|_{1}$.

Proof

Let $\mathbf{v} \in L^{2}(\Omega)^{d}$ and $\phi \in H^{1}(\Omega)$ be such that

$$
\begin{aligned}
\mathbf{v}+\nabla \phi & =\mathbf{u} \\
\nabla \cdot \mathbf{v} & =0,\left.\quad \mathbf{v} \cdot \mathbf{n}\right|_{\partial \Omega}=0
\end{aligned}
$$

It is clear that $\phi$ is solution to the following Poisson problem (up to a constant):

$$
\begin{aligned}
\nabla^{2} \phi & =\nabla \cdot \mathbf{u} \\
\left.\partial_{n} \phi\right|_{\partial \Omega} & =0
\end{aligned}
$$

Owing to the regularizing properties of the Laplacian in smooth domains, we have $\|\phi\|_{2} \leqslant c$ $\|\mathbf{u}\|_{1}$. By definition, $\phi_{h}$ is the Galerkin approximation of $\phi$; hence, we have

$$
\begin{gathered}
\left\|\phi_{h}\right\|_{1} \leqslant c\|\phi\|_{1} \leqslant c^{\prime}\|\mathbf{u}\|_{1} \\
\left\|\phi_{h}-\phi\right\|_{1} \leqslant c h\|\phi\|_{2} \leqslant c^{\prime} h\|\mathbf{u}\|_{1}
\end{gathered}
$$

Since $\widetilde{i_{h}^{\mathrm{T}}}$ is an extension of $i_{h}^{\mathrm{T}}$, we have

$$
\begin{aligned}
\left\|i_{h}^{\mathrm{T}} \mathbf{u}_{h}\right\|_{1} & =\left\|\widetilde{i_{h}^{\mathrm{T}}} \mathbf{u}_{h}\right\|_{1} \\
& =\left\|\widetilde{i_{h}^{\mathrm{T}}} \mathbf{u}-\widetilde{i_{h}^{\mathrm{T}}} \nabla \phi_{h}\right\|_{1} \\
& =\left\|\widetilde{i_{h}^{\mathrm{T}}} \mathbf{u}\right\|_{1}+\left\|\widetilde{i_{h}^{\mathrm{T}}} \nabla\left(\phi_{h}-\phi\right)\right\|_{1}+\left\|\widetilde{i_{h}^{\mathrm{T}}} \nabla \phi\right\|_{1}
\end{aligned}
$$


Then, owing to Lemma 3.1 and estimates (67), (68), we infer

$$
\begin{aligned}
\left\|i_{h}^{\mathrm{T}} \mathbf{u}_{h}\right\|_{1} & \leqslant c\left(\|\mathbf{u}\|_{1}+h^{-1}\left\|\tilde{i_{h}^{\mathrm{T}}} \nabla\left(\phi_{h}-\phi\right)\right\|_{0}+\|\nabla \phi\|_{1}\right) \\
& \leqslant c\left(\|\mathbf{u}\|_{1}+h^{-1}\left\|\nabla\left(\phi_{h}-\phi\right)\right\|_{0}\right) \\
& \leqslant c\|\mathbf{u}\|_{1}
\end{aligned}
$$

This completes the proof.

The pressure estimate follows from the inf-sup condition and can be proved in the same fashion as done by Guermond and Quartapelle [3, Theorem 5.7].

\section{Remark 3.1}

Achdou and Guermond [1] proved that under stronger regularity assumptions on the solution of the continuous problem one can weaken the stability assumption on the time step to $\delta t=O\left(h^{d / 4}\right)$. Because of the enormous technical details involved in this proof we do not reproduce it here in the context of the present scheme.

\section{NUMERICAL RESULTS}

Before presenting the computational results with the first-order scheme, we point out that from practical point of view it is more advantageous to use the second-order version of the scheme discussed in this paper because it yields better accuracy while requiring almost the same resources as the first-order one. It employs a second-order backward difference in time for the time derivative and a second-order extrapolation for the advecting field. The error estimate for such a characteristic scheme using Uzawa iteration for the generalized Stokes problem has been analysed by Boukir et al. [5] yielding a stability condition of the form $\delta t=O\left(h^{d / 6}\right)$. We believe that a similar condition should hold if a second-order projection rather than Uzawa iterations is used, but the proofs are very technical and we do not discuss them here. Such a second-order characteristic scheme has been thoroughly tested numerically by Minev and Ethier [2].

In the remainder of this section, we shall present a brief comparison of the present (firstorder) scheme with the (first-order) scheme of Reference [1]. In the present context, the latter scheme has been implemented slightly differently. To compute the contribution of the convected velocity field $\widetilde{\mathbf{u}}_{h}^{n}$, we first interpolate it on $X_{h}$ and then compute the integrals using a Gauss quadrature. Achdou and Guermond [1] trace the characteristics at the Gauss points in each element thus directly integrating the convected field $\overline{\mathbf{u}}_{h}^{n}$. In both cases the integrals are not exactly computed since the integrands are non-polynomial functions. The computation of these integrals is one of the major problems of the characteristic based methods. The effect of the inexact integration is very hard to evaluate and it is taken into account neither in the present analysis nor the analysis of Achdou and Guermond [1]. Some results for several integration schemes (not including the present one and the one employed in Reference [1]) are presented in Reference [10]. They indicate that some integration schemes can have a destabilizing effect on the entire algorithm. The numerical results with both schemes and the inexact integration as explained above, do not indicate such a behaviour. Note that the present 
integration scheme is potentially cheaper (especially in 3D) than the one used in Reference [1] for it requires to evaluate the foot of only those characteristics that start from a node of a velocity finite element (excluding the boundary nodes). In the implementation of Achdou and Guermond the same should be done for all the Gaussian points in each finite element. In 3D this means at least 15 Gaussian points per element times the number of elements which in many cases is much larger than the number of nodes.

To verify the convergence rate of the two schemes, we have used a 'manufactured' analytic solution of the Navier-Stokes equations (see Reference [11]).

$$
\begin{aligned}
u= & -\left[\mathrm{e}^{x} \sin (y+z)+\mathrm{e}^{z} \cos (x+y)\right] \mathrm{e}^{-d^{2} t} \\
v= & -\left[\mathrm{e}^{y} \sin (z+x)+\mathrm{e}^{x} \cos (y+z)\right] \mathrm{e}^{-d^{2} t} \\
w= & -\left[\mathrm{e}^{z} \sin (x+y)+\mathrm{e}^{y} \cos (z+x)\right] \mathrm{e}^{-d^{2} t} \\
p= & -\frac{1}{2}\left[\mathrm{e}^{2 x}+\mathrm{e}^{2 y}+\mathrm{e}^{2 z}+2 \sin (x+y) \cos (z+x) \mathrm{e}^{y+z}\right. \\
& \left.+2 \sin (y+z) \cos (x+y) \mathrm{e}^{z+x}+2 \sin (z+x) \cos (y+z) \mathrm{e}^{x+y}\right] \mathrm{e}^{-2 t}
\end{aligned}
$$

The Navier-Stokes equations were solved in $\Omega=]-0.5,0.5\left[{ }^{3}\right.$ using a tetrahedral finite element grid, generated as follows. The solution domain was first subdivided into $8^{3}$ uniform subcubes, each of which was further subdivided into five tetrahedra. The resulting grid contained 4401 nodes and 2560 elements. Dirichlet boundary conditions derived from the analytical solution were prescribed on the domain boundary. The Reynolds number was $R e=1$. We integrated the equations with time steps $0.1,0.05,0.025,0.0125$ from time $t=0$ to 3 . The results for the $\ell^{\infty}\left(0,3 ; L^{2}(\Omega)^{d}\right)$ error of both schemes are presented in Figure 1. Although the order of convergence for both schemes are approximately identical, the present scheme produces an error that is about three times smaller. Moreover, the $L^{2}$ norm of the weak divergence of $i_{h}^{\mathrm{T}} \mathbf{u}_{h}$ is about ten times smaller than that of $\tilde{\mathbf{u}}_{h}$.

The next test case is the flow behind a backward facing step which has been thoroughly studied in 2D. We solved the $3 \mathrm{D}$ equations imposing symmetry conditions on the front and back faces of the channel. We used a grid consisting of 22525 nodes and 11800 elements suitably clustered around the step (see Figure 2). One of the most representative and sensitive characteristics of the flow field is the length of the recirculation zone formed behind the step. The recirculation lengths computed with the two schemes at various Reynolds number are compared to available numerical and experimental data in Table I. For the sake of simplicity the recirculation length was not computed from the interpolant of the velocity but we simply recorded the first point in the layer of points neighbouring the wall of the channel (on a distance of 0.1 from the wall), where the sign of the horizontal component of the velocity (alongside the channel) changes. This typically underpredicts the actual length corresponding to the finite-element interpolant. The tests with both schemes, for a given Reynolds number and time step, were run for the same number of time steps. Clearly, the present scheme produces relatively accurate results for larger time steps. The scheme of Reference [1] generally does not reach steady state within the prescribed integration time or even diverges with time steps for which the present scheme works acceptably well. When steady state is not reached with scheme [1] within the prescribed time, we have observed that any further time integration also failed to reach steadiness. We point out that if both schemes reach steadiness and if the 

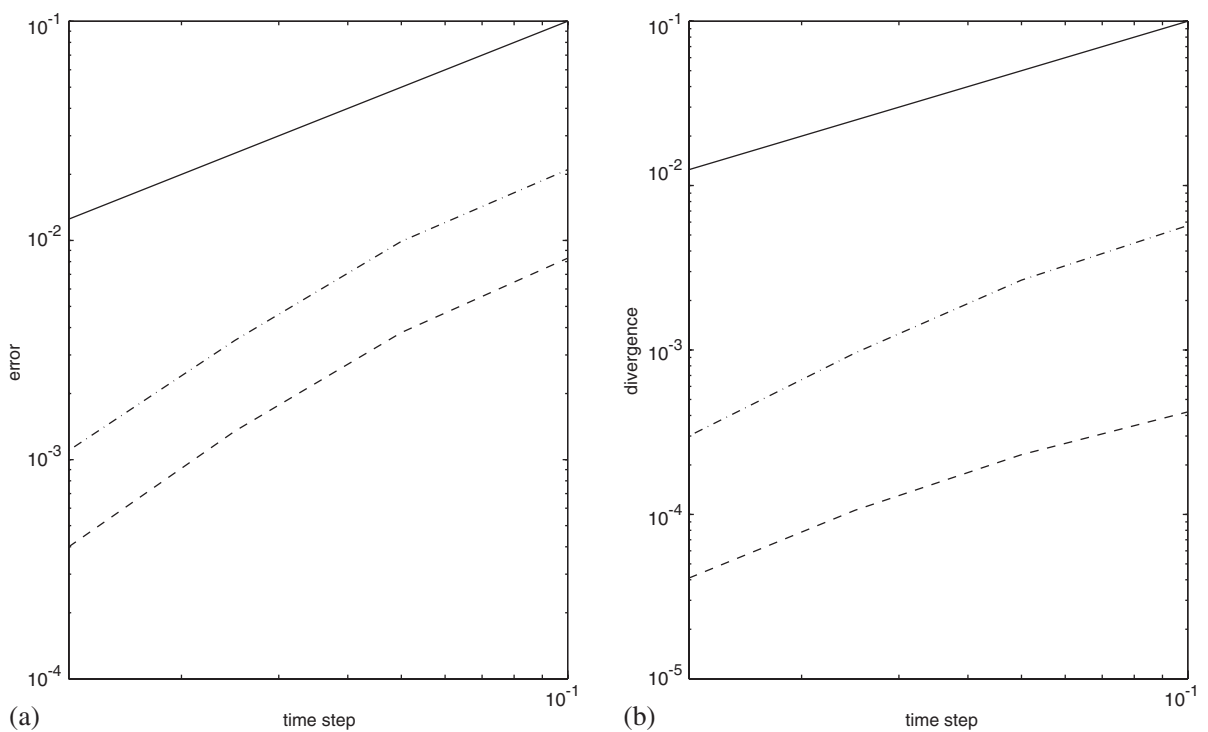

Figure 1. (a) $\ell^{\infty}\left(0,3 ; L^{2}(\Omega)^{d}\right)$ norm of the error in velocity vs. time step: present scheme (dashed line), Achdou and Guermond [1] (dotted line), first-order slope (solid line); and (b) $L^{\infty}\left(0,3 ; L^{2}(\Omega)\right)$ norm of the velocity divergence vs. time step: present scheme (dashed line); Achdou and Guermond [1] (dotted line), first-order slope (solid line).

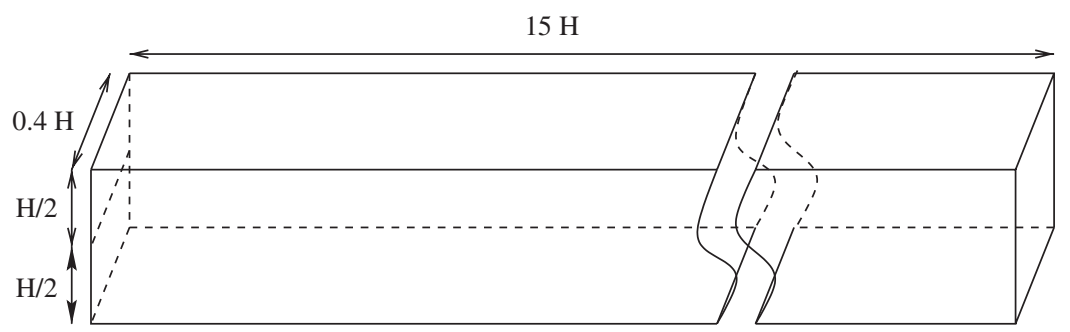

Figure 2.

steady solution is unique, then both schemes must produce the same results since from (21) and (29) we have $\mathbf{u}_{h}^{n+1}=i_{h} \tilde{\mathbf{u}}_{h}^{n+1}$ at steadiness, that is $i_{h}^{\mathrm{T}} \mathbf{u}_{h}^{n+1}=\tilde{\mathbf{u}}_{h}^{n+1}$. The same backward facing step flow is simulated in Reference [2] using the second-order version of the present scheme and the results are in excellent agreement with the available numerical and experimental data.

We finally point out that in some other test cases (a 3D lid-driven cavity for example) the two schemes yielded almost identical results for a wide range of Reynolds numbers. The conclusion from all these numerical results is that if advection is important and the method of characteristics is used, then one should use an advecting velocity field which is as close as possible to a solenoidal field. The present results suggest that for this purpose $i_{h}^{\mathrm{T}} \mathbf{u}_{h}$ is a better candidate than $\tilde{\mathbf{u}}_{h}$. 
Table I. Comparison of the dimensionless length of the recirculation zone for flow over backward-facing step with some experimental or numerical data (for details see Reference [2]). In brackets we provide the time step used to produce the given recirculation length.

\begin{tabular}{cccc}
\hline$R e$ & Available data & Present scheme & Achdou and Guermond scheme \\
\hline \multirow{3}{*}{200} & 5.4 & $5(\delta t=0.2)$ & No st. state $(\delta t=0.2)$ \\
& & $5.1(\delta t=0.1)$ & No st. state $(\delta t=0.1)$ \\
& & $7.1(\delta t=0.05)$ & $5.1(\delta t=0.05)$ \\
400 & 8.5 & $7.9(\delta t=0.4)$ & diverges $(\delta t=0.4)$ \\
& & $8.1(\delta t=0.1)$ & diverges $(\delta t=0.2)$ \\
& & $7.8(\delta t=0.05)$ & No st. state $(\delta t=0.1)$ \\
& & & $7.8(\delta t=0.05)$ \\
\hline
\end{tabular}

\section{ACKNOWLEDGEMENTS}

The first author gratefully acknowledges the support of Centre National de la Recherche Scientifique (CNRS) and that of the Texas Institute for Computational and Applied Mathematics, Austin, Texas, under a TICAM Visiting Faculty Fellowship. The second author would like to acknowledge the support of a research grant from the National Science and Engineering Research Council (NSERC) of Canada.

\section{REFERENCES}

1. Achdou Y, Guermond J-L. Convergence analysis of a finite element projection/Lagrange-Galerkin method for the incompressible Navier-Stokes equations. SIAM Journal on Numerical Analysis 2000; 37(3):799-826.

2. Minev PD, Ethier CR. A semi-implicit projection algorithm for the Navier-Stokes equations with application to flows in complex geometries. In Notes on Numerical Fluid Mechanics, vol. 73, Griebel et al. (eds). Vieweg: Braunschweig/Wiesbaden, 1999; 223-231.

3. Guermond J-L, Quartapelle L. On the approximation of the unsteady Navier-Stokes equations by finite element projection methods. Numerische Mathematik 1998; 80:207-238.

4. Pironneau O. On the transport-diffusion algorithm and its applications to the Navier-Stokes equations. Numerische Mathematik 1982; 38:309-332.

5. Boukir K, Maday Y, Metivet B, Razafindrakoto E. A high order characteristics/finite element method for the incompressible Navier-Stokes equations. International Journal for Numerical Methods in Fluids 1997; 25:1421-1454.

6. Douglas J, Russell TF. Numerical methods for convection dominated diffusion problems based on combining the method of characteristics with finite element methods or finite difference method. SIAM Journal on Numerical Analysis 1982; 19:871-885. Stepleman RS (eds), Advances in Computational Methods for P.D.E. 1981; $28-36$.

7. Süli E. Convergence and non-linear stability of the Lagrange-Galerkin method for the Navier-Stokes equations. Numerische Mathematik 1988; 53:459-483.

8. Gresho PM, Chan ST. On the theory of semi-implicit projection methods for viscous incompressible flow and its implementation via a finite element method that also introduces a nearly consistent mass matrix. Part I and Part II. International Journal for Numerical Methods in Fluids 1990; 11:587-620.

9. Ciarlet PG. The Finite Element Method for Elliptic Problems. North-Holland: Amsterdam, 1978.

10. Morton KW, Priestley A, Surli E. Stability of the Lagrange-Galerkin method with non-exact integration. Mathematical Modelling in Numerical Analysis 1988; 22:625-653.

11. Ethier CR, Steinman DA. Exact fully 3-D Navier-Stokes solution for benchmarking. International Journal for Numerical Methods in Fluids 1994; 19:369-375. 